\title{
An Extension of the Picard Theorem to Fractional Differential Equations with a Caputo-Fabrizio Derivative
}

\author{
H. R. Marasi $\mathbb{D}^{1}{ }^{1}$ A. Soltani Joujehi, ${ }^{1}$ and H. Aydi $\mathbb{D}^{2,3,4}$ \\ ${ }^{1}$ Department of Applied Mathematics, Faculty of Mathematical Sciences, University of Tabriz, Tabriz, Iran \\ ${ }^{2}$ Université de Sousse, Institut Supérieur d'Informatique et des Techniques de Communication, H. Sousse 4000, Tunisia \\ ${ }^{3}$ China Medical University Hospital, China Medical University, Taichung 40402, Taiwan \\ ${ }^{4}$ Department of Mathematics and Applied Mathematics, Sefako Makgatho Health Sciences University, Ga-Rankuwa, South Africa
}

Correspondence should be addressed to H. R. Marasi; marasi@tabrizu.ac.ir and H. Aydi; hassen.aydi@isima.rnu.tn

Received 26 November 2020; Revised 14 February 2021; Accepted 6 March 2021; Published 15 March 2021

Academic Editor: Ali Aral

Copyright (c) 2021 H. R. Marasi et al. This is an open access article distributed under the Creative Commons Attribution License, which permits unrestricted use, distribution, and reproduction in any medium, provided the original work is properly cited.

In this paper, we consider fractional differential equations with the new fractional derivative involving a nonsingular kernel, namely, the Caputo-Fabrizio fractional derivative. Using a successive approximation method, we prove an extension of the Picard-Lindelöf existence and uniqueness theorem for fractional differential equations with this derivative, which gives a set of conditions, under which a fractional initial value problem has a unique solution.

\section{Introduction}

Due to the demonstrated applications of fractional operators in various and widespread fields of many sciences, such as mathematics, physics, chemistry, engineering, and statistics [1-4], various operators of a fractional calculus have been found to be remarkably popular for modelling of numerous varied problems in these sciences. We mention here some of these definitions, such as Riemann-Liouville, Hadamard, Grünwald-Letnikov, Weyl, Riesz, Erdélyi-Kober, and Caputo. Compared with an integer order, a significant feature of a fractional order differential operator appeared in its hereditary property. In other words, when we describe a process by a fractional operator, we predict the future state by its current as well as its past states. Therefore, the memory and hereditary properties of materials and systems can be intervened in the modeling of a process by making use of differential equations of an arbitrary order. So, in recent years, fractional differential equations have been paid a great interest and also have appeared in new areas for applications of initial and boundary value problems of such equations. The Riemann-Liouville definition for the fractional derivative is one of the most widely used definitions and has many applications. But this definition had its drawbacks, such as the fact that the derivative of a constant function is not zero, and in practical examples, we need the value of fractional derivatives as initial values. The Caputo fractional derivative does not have the above weaknesses and is believed to be one of the most efficient definitions of fractional derivative applied in many areas of science and engineering.

However, the new definition suggested by Caputo and Fabrizio [5], which has all the characteristics of the old definitions, assumes two different representations for the temporal and spatial variables. In fact, they claimed that the classical definition given by Caputo appears to be particularly convenient for mechanical phenomena, related with plasticity, fatigue, damage, and with electromagnetic hysteresis. When these effects are not present, it seems more appropriate to use the new Caputo-Fabrizio operator.

The main advantage of the Caputo-Fabrizio approach is that the boundary conditions of the fractional differential equations with Caputo-Fabrizio derivatives admit the same form as for the integer-order differential equations. On the other hand, the Caputo-Fabrizio fractional derivative has many significant properties, such as its ability in describing matter heterogeneities and configurations with different scales [6-8]. Therefore, there are some certain phenomena that cannot be well-modeled using the Riemann-Liouville, 
Caputo, or other standard fractional operators [5, 9-13]. For an example, in issues related to material heterogeneities, we encounter some problems that are not well described by the above fractional operators. Also, later, some other definitions with a nonsingular kernel, such as the Atangana-Baleanu [6] fractional derivative, were defined.

Many researchers have shared their contributions to obtain properties of many models with new and old definitions of fractional derivatives. In [14], we have the analytic solutions of a viscous fluid with the Caputo and CaputoFabrizio fractional derivatives. In [15], the authors used the fractional derivative with a nonsingular kernel to model a Maxwell fluid and found semianalytical solutions. In [16], we found a comparison approach of two latest fractional derivatives models, namely, Atangana-Baleanu and CaputoFabrizio, for a generalized Casson fluid and obtained exact solutions. In [17-19], the authors also used the CaputoFabrizio fractional derivative to model some important examples.

Due to the abovementioned applications, the existence of solutions for nonlinear differential equations is an attractive research topic and has been studied using different techniques of nonlinear analysis [20-23]. One of the most important theorems in ordinary differential equations is Picard's existence and uniqueness theorem. This theorem, which is applied on first-order ordinary differential equations, can be generalized to establish existence and uniqueness results for both higher-order ordinary differential equations and for systems of differential equations. This theorem is a good introduction to the broad class of existence and uniqueness theorems that are based on fixed-point techniques [24-30].

In this paper, we obtain an extension of Picard's theorem for differential equations with the Caputo-Fabrizio fractional derivative. This theorem provides conditions for which a fractional initial value problem involving the CaputoFabrizio derivative has a unique solution. On the other hand, the proof of this extension of Picard's theorem provides a way of constructing successive approximations to the solution.

\section{Preliminaries}

In this section, we recall some notations and definitions which are needed throughout this paper. Further, some lemmas and theorems are stated as preparations for the main results. First, in the following, we provide some basic concepts and definitions in connection with the new CaputoFabrizio derivative.

The well-known left-sided Caputo fractional derivative ${ }^{C} D_{0^{+}, t}^{\alpha}$ of a function $f(x) \in H^{1}(0, b)$ with $0<\alpha<1$, is defined by

$$
{ }^{C} D_{0^{+}, t}^{\alpha} g(t)=\frac{1}{\Gamma(1-\alpha)} \int_{0}^{t} f^{\prime}(s)(t-s)^{-\alpha} d s
$$

In [5], Caputo and Fabrizio proposed the new operator by replacing the singular kernel $(x-t)^{-\alpha}$ with $e^{-\alpha(x-t) /(1-\alpha)}$ and $1 / \Gamma(1-\alpha)$ with $N(\alpha) /(1-\alpha)$ in the Caputo definition to obtain the following definition.

Definition 1. Let $g$ be a given function in $H^{1}(a, b)$. The Caputo-Fabrizio derivative of fractional order $\alpha \in[0,1]$ is defined as

$$
{ }_{a}^{C F} D_{t}^{\alpha}(g(t))=\left(\frac{N(\alpha)}{1-\alpha}\right) \int_{a}^{t} g^{\prime}(x) \exp \left[-\alpha \frac{t-x}{1-\alpha}\right] d x
$$

where $N(\alpha)$ is a normalization function [5]. Also, if a certain function $g$ does not satisfy in the restriction $g \in H^{1}(a, b)$, then its fractional derivative is redefined as

$$
{ }_{a}^{C F} D_{t}^{\alpha}(g(t))=\frac{\alpha N(\alpha)}{1-\alpha} \int_{a}^{t}(g(t)-g(x)) \exp \left[-\alpha \frac{t-x}{1-\alpha}\right] d x .
$$

Clearly, as mentioned in [5], if one sets $\sigma=(1-\alpha) / \alpha \in[$ $0, \infty]$ and $\alpha=1 /(1+\sigma) \in[0,1]$, then the Caputo-Fabrizio definition becomes

$$
{ }_{a}^{C F} D_{t}^{\alpha}(g(t))=\frac{N(\sigma)}{\sigma} \int_{a}^{t} g^{\prime}(x) \exp \left[-\frac{t-x}{\sigma}\right] d x
$$

where $N(0)=N(\infty)=1$, and

$$
\lim _{\sigma \rightarrow 0} \exp \left[-\frac{t-x}{\sigma}\right]=\delta(x-t)
$$

Also, the fractional derivative of order $(n+\alpha)$ when $n \geq 1$ and $\alpha \in[0,1]$ is defined by the following

$$
{ }_{a}^{C F} D_{t}^{(\alpha+n)}(g(t))={ }_{a}^{C F} D_{t}^{(\alpha)}\left(D_{t}^{(n)} g(t)\right)
$$

Definition 2. Let $g \in H^{1}(a, b)$, then its fractional integral of an arbitrary order is defined as follows:

$$
{ }_{a}^{C F} I_{t}^{\alpha}(g(t))=\frac{2(1-\alpha)}{(2-\alpha) N(\alpha)} g(t)+\frac{2 \alpha}{(2-\alpha) N(\alpha)} \int_{a}^{t} g(s) d s, \quad t \geq 0 .
$$

It is clear, in view of the above definition, that the $\alpha$ th Caputo-Fabrizio derivative of a function $g$ is average between $g$ and its first-order integral. Therefore,

$$
\frac{2(1-\alpha)}{(2-\alpha) N(\alpha)}+\frac{2 \alpha}{(2-\alpha) N(\alpha)}=1
$$

So, we arrive at the following

$$
N(\alpha)=\frac{2}{2-\alpha}, \quad 0 \leq \alpha \leq 1
$$

The Laplace transform of the Caputo-Fabrizio derivative 
is

$$
L\left\{{ }^{C F} D_{t}^{\alpha}(g(t))\right\}=\frac{s L\{g(t)\}-g(0)}{(1-\alpha) s+\alpha} .
$$

Theorem 3 (Picard theorem [31]). Let D be an open set in ( $t, x)$-space. Let $\left(t_{0}, x^{0}\right) \in D$ and $a$ and $b$ be positive constants such that the set

$$
R=\left\{(t, x)|| t-t_{0}|\leq a,| x-x_{0} \mid \leq b\right\}
$$

is contained in D. Suppose that the function $g$ is defined, continuous on $D$, and satisfies a Lipschitz condition with respect to $x$ in $R$. Let

$$
\begin{aligned}
& M=\max _{(t, x) \in R}|g(t, x)|, \\
& A=\min \left\{a, \frac{b}{M}\right\} .
\end{aligned}
$$

Then, the following initial value problem

$$
x^{\prime}=g(t, x), x\left(t_{0}\right)=x^{0}
$$

has a unique solution, $x(t)$, on the interval $\left(t_{0}-A, t_{0}+A\right)$. For this solution in the domain $\left(t_{0}-A, t_{0}+A\right)$, we have

$$
\left|x(t)-x^{0}\right| \leq M A
$$

Note that by the mean-value theorem, the Lipschitz condition will be satisfied if we have $|(\partial / \partial x) g(t, x)| \leq K$.

\section{Extension of Picard Theorem}

Picard's Theorem 3 guarantees the existence and uniqueness of the solution of the following initial value problem of firstorder differential equations:

$$
\begin{aligned}
\frac{d y}{d t} & =f(t, y(t)) t \geq t_{0}, \\
y\left(t_{0}\right) & =y_{0} .
\end{aligned}
$$

In proving this theorem, the solution is obtained by the well-known successive approximations method (Picard-Lindelöf method) [31]. In this method, the approximate solution for solving (15) is defined by

$$
y_{k+1}=y_{0}+\int_{t_{0}}^{t} f\left(s, y_{k}(s)\right) d s, \quad k \in N .
$$

By continuing this process, when $k \rightarrow \infty$, the exact solution is obtained. In practice, the exact solution is approximated for a sufficient large $k$ by $y_{k}$.

In this section, we consider the following differential equation

$$
{ }_{0}^{C F} D_{t}^{\alpha} u(t)=g(t, u)
$$

such that $t \in J=[0,1]$, with the initial condition $u(0)=u_{0}$, where ${ }_{0}^{C F} D_{t}^{\alpha}$ denotes the fractional Caputo-Fabrizio derivative. We extend Picard's theorem to this problem, and by the successive approximation method, an iterative process is provided to obtain the solution. We state the following generalized Picard existence and uniqueness theorem.

Theorem 4. Suppose that the function $g$ is defined, continuous on an open set $\Omega$ in $(t, u)$-space, and satisfies

$$
|g(t, u)-g(t, v)| \leq k|u-v|, \quad 0<k<1 .
$$

Let $M=\max _{t \in J}|g(t, u)|$. Then, the fractional differential equation (18) has a unique solution such that $u(0)=u_{0}$.

To prove the theorem, first, we need to establish the following lemma.

Lemma 5. The function $u(t)$ is the solution of (18) under the initial condition $u(0)=u_{0}$ if and only if it satisfies the following integral equation:

$$
\begin{aligned}
u(t)= & u_{0}+\frac{2(1-\alpha)}{(2-\alpha) N(\alpha)} g(t, u(t)) \\
& +\frac{2 \alpha}{(2-\alpha) N(\alpha)} \int_{0}^{t} g(s, u(s)) d s .
\end{aligned}
$$

Proof. If $u(t)$ is a solution of (18), then taking the fractional integral of order $\alpha$, we obtain (20). The second part of the theorem comes from differentiating equation (20).

In the reminder of the proof, using the successive approximation method, we show that the sequence defined by

$$
\begin{aligned}
u_{0}(t)= & u_{0}, \\
u_{1}(t)= & u_{0}+\frac{2(1-\alpha)}{(2-\alpha) N(\alpha)} g\left(t, u_{0}\right) \\
& +\frac{2 \alpha}{(2-\alpha) N(\alpha)} \int_{0}^{t} g\left(s, u_{0}\right) d s, \\
\vdots & \\
u_{m}(t)= & u_{0}+\frac{2(1-\alpha)}{(2-\alpha) N(\alpha)} g\left(t, u_{m-1}(t)\right) \\
& +\frac{2 \alpha}{(2-\alpha) N(\alpha)} \int_{0}^{t} g\left(s, u_{m-1}(s)\right) d s
\end{aligned}
$$

converges to a function, which is a solution of (20), and then we show that this solution is unique.

Lemma 6. For each $m$, the function $u_{m}(t)$ is defined, continuous on $J$ and satisfies

$$
\left|u_{m}(t)-u_{0}\right| \leq M
$$


Proof. We prove the lemma by induction. Since

$$
\begin{aligned}
\left|u_{1}(t)-u_{0}(t)\right| \leq & \frac{2(1-\alpha)}{(2-\alpha) N(\alpha)}\left|g\left(t, u_{0}(t)\right)\right| \\
& +\frac{2 \alpha}{(2-\alpha) N(\alpha)} \int_{0}^{t}\left|g\left(s, u_{0}(s)\right)\right| d s \\
\leq & \frac{2(1-\alpha)}{(2-\alpha) N(\alpha)} M+\frac{2 \alpha}{(2-\alpha) N(\alpha)} M \int_{0}^{t} \\
& \cdot d s \leq \frac{2(1-\alpha)}{(2-\alpha) N(\alpha)} M \\
& +\frac{2 \alpha}{(2-\alpha) N(\alpha)} M=M
\end{aligned}
$$

the result is obviously true for $m=0$. Let us suppose that for $t \in J$,

$$
\left|u_{m}(t)-u_{0}\right| \leq M
$$

This yields that $f\left(t, u_{m}(t)\right)$ is defined on $J$, and since $f(t$ , $\left.u_{m}(t)\right)$ is continuous at $t$, one asserts that

$$
\begin{aligned}
u_{m+1}(t)= & u_{0}+\frac{2(1-\alpha)}{(2-\alpha) N(\alpha)} g\left(t, u_{m}(t)\right) \\
& +\frac{2 \alpha}{(2-\alpha) N(\alpha)} \int_{0}^{t} g\left(s, u_{m}(s)\right) d s
\end{aligned}
$$

is defined and continuous. Indeed, we have

$$
\begin{aligned}
& \left|u_{m+1}(t)-u_{0}\right| \leq \frac{2(1-\alpha)}{(2-\alpha) N(\alpha)}\left|g\left(t, u_{m}(t)\right)\right| \\
& +\frac{2 \alpha}{(2-\alpha) N(\alpha)} \int_{0}^{t}\left|g\left(s, u_{m}(s)\right)\right| d s \leq \frac{2(1-\alpha)}{(2-\alpha) N(\alpha)} M \\
& \quad+\frac{2 \alpha}{(2-\alpha) N(\alpha)} M \int_{0}^{t} d s=M .
\end{aligned}
$$

Lemma 7. The sequence $\left\{u_{m}(t)\right\}$ converges uniformly on $J$ to a continuous function $u(t)$.

Proof. It is obvious that the convergence of the series

$$
u_{0}(t)+\sum_{n=0}^{\infty}\left[u_{n+1}(t)-u_{n}(t)\right]
$$

yields convergence of the sequence $\left\{u_{m}(t)\right\}$. For $t \in J$, let us denote

$$
\begin{aligned}
& d_{n}(t)=\left|u_{n+1}(t)-u_{n}(t)\right| \\
& F_{n}(t)=g\left(t, u_{n+1}(t)\right)-g\left(t, u_{n}(t)\right)
\end{aligned}
$$

Then, for each $n$, one has

$$
\begin{aligned}
& d_{n}(t) \leq \frac{2(1-\alpha)}{(2-\alpha) N(\alpha)}\left|F_{n}(t)\right| \\
& \quad+\frac{2 \alpha}{(2-\alpha) N(\alpha)} \int_{0}^{t}\left|F_{n}(s)\right| d s \\
& \leq \frac{2(1-\alpha)}{(2-\alpha) N(\alpha)} k\left|u_{n}(t)-u_{n-1}(t)\right| \\
& \quad+\frac{2 \alpha}{(2-\alpha) N(\alpha)} k \int_{0}^{t}\left|u_{n}(s)-u_{n-1}(s)\right| d s \\
& =\frac{2(1-\alpha)}{(2-\alpha) N(\alpha)} k d_{n-1}(t)+\frac{2 \alpha}{(2-\alpha) N(\alpha)} k \int_{t_{0}}^{t} \\
& \quad \cdot d_{n-1}(s) d s=k_{0}^{C F} I_{t}^{\alpha} d_{n-1}(t),
\end{aligned}
$$

where $k$ is the Lipschitz constant of $g$ and $0<k<1$. Now, we show that for each $n$, we have

$$
d_{n}(t) \leq M k^{n}
$$

From Lemma 6, we have

$$
d_{0}(t)=\left|u_{1}(t)-u_{0}(t)\right| \leq M
$$

By induction, let $d_{n}(t) \leq M k^{n}$. Then, from (29) and (8), one writes

$$
\begin{aligned}
& d_{n+1}(t) \leq k_{0}^{C F} I_{t}^{\alpha} d_{n}(t)=\frac{2(1-\alpha)}{(2-\alpha) N(\alpha)} k d_{n}(t) \\
& +\frac{2 \alpha}{(2-\alpha) N(\alpha)} k \int_{t_{0}}^{t} d_{n}(s) d s \leq \frac{2(1-\alpha)}{(2-\alpha) N(\alpha)} k M k^{n} \\
& +\frac{2 \alpha}{(2-\alpha) N(\alpha)} k M k^{n} \int_{0}^{t} d s \leq \frac{2(1-\alpha)}{(2-\alpha) N(\alpha)} M k^{n+1} \\
& +\frac{2 \alpha}{(2-\alpha) N(\alpha)} M k^{n+1}=M k^{n+1} .
\end{aligned}
$$

Therefore,

$$
\sum_{n=0}^{\infty} d_{n}(t) \leq M \sum_{n=0}^{\infty} k^{n}
$$

Since $0<k<1$, the uniform convergence of (27) follows from the Weierstrass test or by a simple comparison test.

Lemma 8. The function $u(t)$ is satisfied in (18), and we have $u(0)=u_{0}$.

Proof. First, let us show that $\left|u(t)-u_{0}\right|$ is bounded. That is,

$$
\left|u(t)-u_{0}\right|<B
$$

for some constant $B$. We can deduce that $g(t, u(t))$ is defined for $t \in J$. For $t \in J$ and $\varepsilon>0$ and for a sufficiently large $m$, one 
has

$$
\left|u(t)-u_{0}\right| \leq\left|u(t)-u_{m}(t)\right|+\left|u_{m}(t)-u_{0}\right| \leq \varepsilon+M<B .
$$

Then, by the Lipschitz condition of $g$, we have

$$
\begin{aligned}
& \left|\int_{0}^{t} g(s, u(s))-g\left(s, u_{m}(s)\right) d s\right| \leq \int_{0}^{t} \mid g(s, u(s)) \\
& \quad-g\left(s, u_{m}(s)\right)\left|d s \leq k \int_{0}^{t}\right| u(s)-u_{m}(s) \mid d s \leq k \varepsilon .
\end{aligned}
$$

Therefore, $\lim _{m \rightarrow \infty} \int_{0}^{t} g\left(s, u_{m}(s)\right)=\int_{0}^{t} g(s, u(s)) d s$. Now, by taking the limit with respect to $m$ on both sides of the following equation

$$
\begin{aligned}
u_{m}(t)= & u_{0}+\frac{2(1-\alpha)}{(2-\alpha) N(\alpha)} g\left(t, u_{m-1}(t)\right) \\
& +\frac{2 \alpha}{(2-\alpha) N(\alpha)} \int_{0}^{t} g\left(s, u_{m-1}(s)\right) d s
\end{aligned}
$$

we obtain

$$
\begin{aligned}
u(t)= & u_{0}+\frac{2(1-\alpha)}{(2-\alpha) N(\alpha)} g(t, u(t)) \\
& +\frac{2 \alpha}{(2-\alpha) N(\alpha)} \int_{0}^{t} g(s, u(s)) d s
\end{aligned}
$$

Now, we prove the uniqueness of the solution.

Lemma 9. The solution $u(t)$ of the integral equation (7) satisfying the condition $u\left(t_{0}\right)=u_{0}$, is the unique solution of (18) with this initial condition.

Proof. Suppose that there exist two solutions $u_{1}(t)$ and $u_{2}(t)$ of the integral equation (7) on $J$ subject to the condition $u_{1}$ $\left(t_{0}\right)=u_{2}\left(t_{0}\right)=u_{0}$. First, since $u_{1}(t)$ and $u_{2}(t)$ are continuous functions, there exists a constant $B>0$ such that in the closed interval $J$, we have

$$
\left|u_{1}(t)-u_{2}(t)\right|<B
$$

Let us suppose that for each positive integer $m$,

$$
\left|u_{1}(t)-u_{2}(t)\right|<k^{m} B
$$

Then, from (7), we have $\left|u_{1}(t)-u_{2}(t)\right|<k^{m+1} B$. Therefore, by induction, $\left|u_{1}(t)-u_{2}(t)\right|$ is less than each term of the convergent geometric series of $B /(1-k)$. This yields that for each $\varepsilon,\left|u_{1}(t)-u_{2}(t)\right|<\varepsilon$, and therefore, we have $u_{1}(t)$ $=u_{2}(t)$.

By proving the above lemma, the proof of Theorem 3 is completed. Note that the iterative process (21) provides a constructive approach to obtain the solution. We describe the following simple example where the hypotheses of Theo- rem (4) hold:

$$
\begin{aligned}
{ }_{0}^{C F} D_{t}^{\alpha} u(t) & =\frac{1}{u+1}, \\
u(0) & =0 .
\end{aligned}
$$

By assuming $C=2(1-\alpha) /(2-\alpha) N(\alpha)$ and $D=2 \alpha /(2-$ $\alpha) N(\alpha)$, the results of using (21) are as follows:

$$
\begin{aligned}
u_{0}(t)= & 0, \\
u_{1}(t)= & C+D t, \\
u_{2}(t)= & \frac{C}{D t+C+1}+\ln (D t+C+1), \\
u_{3}(t)= & \frac{C}{C /(D t+C+1)+\ln (D t+C+1)+1} \\
& +D \int_{0}^{t} \frac{1}{C /(D s+C+1)+\ln (D s+C+1)+1} .
\end{aligned}
$$

To ensure the results, let us choose $\alpha=1$. In this case, it is easy to show that the obtained sequence $0, t, \ln$ $(t+1), \cdots$ converges to the exact solution $u(t)=\sqrt{2 t+1}-1$.

\section{Conclusion}

By Picard's theorem, we can study the existence and uniqueness of a solution of first-order differential equations. Also, this theorem can be applied to ensure the existence of a unique solution of higher-order ordinary differential equations and for systems of differential equations. On the other hand, this theorem is an essential tool in fixed-point theory. Therefore, a generalization of this theorem for fractional differential equations would be interesting. In this paper, we proved an extension of this theorem to the initial value problems of fractional ordinary differential equations with the Caputo-Fabrizio derivative, and by the successive approximation method, an iterative process was provided to obtain the solution.

\section{Data Availability}

No data were used to support this study.

\section{Conflicts of Interest}

The authors declare no conflict of interest.

\section{Authors' Contributions}

All authors contributed equally and significantly in writing this article. 


\section{References}

[1] A. A. Kilbas, H. M. Sirvastava, and J. J. Trujillo, Theory and Applications of Fractional Differential Equations, Elsevier, Amsterdam, The Netherlands, 2006.

[2] I. Podlubny, "Geometric and physical interpretation of fractional integration and fractional differentiation," Fractional Calculus and Applied Analysis, vol. 5, no. 4, pp. 367-386, 2002.

[3] K. S. Miller and B. Ross, An Introduction to the Fractional Calculus and Fractional Differential Equations, Wiley, New York, 1993.

[4] M. Caputo, "Linear models of dissipation whose Q is almost frequency independent-II," Geophysical Journal International, vol. 13, no. 5, pp. 529-539, 1967.

[5] M. Caputo and Fabrizio, "A new definition of fractional derivative without singular kernel," Progress in Fractional Differentiation and Applications, vol. 1, pp. 73-85, 2015.

[6] A. Atangana and D. Baleanu, "New fractional derivatives with nonlocal and non-singular kernel: theory and application to heat transfer model," Thermal Science, vol. 20, no. 2, pp. 763-769, 2016.

[7] J. Losada and J. J. Nieto, "Properties of the new fractional derivative without singular kernel," Progress in Fractional Differentiation and Applications, vol. 1, pp. 87-92, 2015.

[8] A. Atangana and B. S. T. Alkahtani, "Analysis of the KellerSegel model with a fractional derivative without singular kernel," Entropy, vol. 17, no. 12, pp. 4439-4453, 2015.

[9] H. R. Marasi and S. Karimi, "Convergence of the variational iteration method for solving fractional Klein-Gordon equation," Journal of Mathematical and Computational Science, vol. 4, no. 2, pp. 257-266, 2014.

[10] H. R. Marasi and M. Nikbakht, "The Adomian decomposition method for boundary eigenvalue problems," Australian Journal of Basic and Applied Sciences, vol. 5, no. 12, pp. 21062111, 2011.

[11] A. Alshabanat, M. Jleli, S. Kumar, and B. Samet, "Generalization of Caputo-Fabrizio fractional derivative and applications to electrical circuits," Frontiers of Physics, vol. 8, 2020.

[12] F. E. Bouzenna, M. T. Meftah, and M. Difallah, "Application of the Caputo-Fabrizio derivative without singular kernel to fractional Schrodinger equations," Pramana, vol. 94, article 92, 2020.

[13] K. Shah, F. Jarad, and T. Abdeljawad, "On a nonlinear fractional order model of dengue fever disease under CaputoFabrizio derivative," Alexandria Engineering Journal, vol. 59, no. 4, pp. 2305-2313, 2020.

[14] N. A. Shah, M. A. Imran, and F. Miraj, "Exact solutions of time fractional free convection flows of viscous fluid over an isothermal vertical plate with Caputo and Caputo-Fabrizio derivatives," Journal of Prime Research in Mathematics, vol. 13, pp. 56-74, 2017.

[15] M. A. Imran, M. B. Riaz, N. A. Shah, and A. A. Zafar, "Boundary layer flow of MHD generalized Maxwell fluid over an exponentially accelerated infinite vertical surface with slip and Newtonian heating at the boundary," Results in Physics, vol. 8, pp. 1061-1067, 2018.

[16] N. A. Sheikh, F. Ali, M. Saqib, I. Khan, and S. A. A. Jan, "A comparative study of Atangana-Baleanu and CaputoFabrizio fractional derivatives to the convective flow of a generalized Casson fluid," The European Physical Journal Plus, vol. 132, article 54, 2017.
[17] I. Khan, N. A. Shah, Y. Mashud, and D. Vieru, "Heat transfer analysis in a Maxwell fluid over an oscillating vertical plate using fractional Caputo-Fabrizio derivatives," The European Physical Journal Plus, vol. 132, article 192, 2017.

[18] H. Aydi, M. Jleli, and B. Samet, "On positive solutions for a fractional thermostat model with a convex-concave source term via $\psi$-Caputo fractional derivative," Mediterranean Journal of Mathematics, vol. 17, no. 1, article 16, 2020.

[19] Q. Rubbaba, M. Nazeerb, F. Ahmad, Y. M. Chu, M. I. Khan, and S. Kadry, "Numerical simulation of advection-diffusion equation with Caputo-Fabrizio time fractional derivative in cylindrical domains: applications of pseudo-spectral collocation method," Alexandria Engineering Journal, vol. 60, no. 1, pp. 1731-1738, 2021.

[20] C. Ravichandran, K. Logeswari, and F. Jarad, "New results on existence in the framework of Atangana-Baleanu derivative for fractional integro-differential equations," Chaos, Solitons \& Fractals, vol. 125, pp. 194-200, 2019.

[21] K. Jothimani, K. Kaliraj, Z. Hammouch, and C. Ravichandran, "New results on controllability in the framework of fractional integrodifferential equations with nondense domain," The European Physical Journal Plus, vol. 134, no. 9, p. 441, 2019.

[22] N. Valliammal, C. Ravichandran, and K. S. Nisar, "Solutions to fractional neutral delay differential nonlocal systems," Chaos, Solitons \& Fractals, vol. 138, article 109912, 2020.

[23] V. Gupta, F. Jarad, N. Valliammal, C. Ravichandran, and K. S. Nisar, "Existence and uniqueness of solutions for fractional nonlinear hybrid impulsive system," Numerical Methods for Partial Differential Equations, pp. 1-13, 2020.

[24] E. Ameer, H. Aydi, M. Arshad, and M. De la Sen, "Hybrid Ćirić type graphic $(Y, \Lambda)$-contraction mappings with applications to electric circuit and fractional differential equations," Symmetry, vol. 12, no. 3, p. 467, 2020.

[25] H. R. Marasi and H. Aydi, "Existence and uniqueness results for two-term nonlinear fractional differential equations via a fixed point technique," Journal of Mathematics, vol. 2021, Article ID 6670176, 7 pages, 2021.

[26] H. A. Hammad, H. Aydi, and N. Mlaiki, "Contributions of the fixed point technique to solve the $2 \mathrm{D}$ Volterra integral equations, Riemann-Liouville fractional integrals, and AtanganaBaleanu integral operators," Advances in Difference Equations, vol. 2021, Article ID 97, 2021.

[27] H. A. Hammad, H. Aydi, and Y. U. Gaba, "Exciting fixed point results on a novel space with supportive applications," Journal of Function Spaces, vol. 2021, Article ID 6613774, 12 pages, 2021.

[28] H. R. Marasi, H. Afshari, and C. B. Zhai, "Some existence and uniqueness results for nonlinear fractional partial differential equations," The Rocky Mountain Journal of Mathematics, vol. 47, no. 2, pp. 571-585, 2017.

[29] H. Afshari, H. R. Marasi, and H. Aydi, "Some existence and uniqueness for positive solutions for boundary value problems of fractional differential equations," Univerzitet u Nišu, vol. 31, no. 9, pp. 2675-2682, 2017.

[30] C. B. Zhai, L. Zhao, S. Li, and H. R. Marasi, "On some properties of positive solutions for a third-order three-point boundary value problem with a parameter," Advances in Difference Equations, vol. 2017, Article ID 187, 2017.

[31] W. G. Kelley and A. C. Peterson, The Theory of Differential Equations, Springer, 2010. 